\title{
Predictive value of the National Institutes of Health Stroke Scale and the Mini-Mental State Examination for neurologic outcome after coronary artery bypass graft surgery
}

\author{
Nancy A. Nussmeier, MD, ${ }^{\mathrm{a}}$ Yinghui Miao, MD, MPH, ${ }^{\mathrm{b}}$ Gary W. Roach, MD, ${ }^{\mathrm{c}}$ Richard L. Wolman, MD, ${ }^{\mathrm{d}}$ \\ Christina Mora-Mangano, MD, ${ }^{\mathrm{e}}$ Mark Fox, MD, ${ }^{\mathrm{f}}$ Andrea Szekely, MD, ${ }^{\mathrm{g}}$ Concezione Tommasino, MD, ${ }^{\mathrm{h}}$ \\ Nanette M. Schwann, MD, ${ }^{\mathrm{i}}$ and Dennis T. Mangano, $\mathrm{MD}, \mathrm{PhD},{ }^{\mathrm{b}}$ for the Investigators of the Ischemia and \\ Education Foundation and the Multicenter Study of Perioperative Ischemia Research Group*
}

\begin{abstract}
Objective: We intended to define the role of the National Institutes of Health Stroke Scale and the Mini-Mental State Examination in identifying adverse neurologic outcomes in a large international sample of patients undergoing cardiac surgery.
\end{abstract}

Methods: We evaluated 4707 patients undergoing cardiac surgery with cardiopulmonary bypass at 72 centers in 17 countries between November 1996 and June 2000. Prespecified overt neurologic outcomes were categorized as type I (clinically diagnosed stroke, transient ischemic attack, encephalopathy, or coma) or type II (deterioration of intellectual function). The National Institutes of Health Stroke Scale and Mini-Mental State Examination were administered preoperatively and on postoperative day 3, 4, or 5. Receiver operating characteristic curves were plotted to determine the predictive value of worsening in National Institutes of Health Stroke Scale and MiniMental State Examination scores with respect to type I and II outcomes.

Results: The receiver operating characteristic area under the curve for changes in National Institutes of Health Stroke Scale score $(\mathrm{n}=4620)$ was 0.89 for type I outcomes and 0.66 for type II outcomes. A 1-point worsening in National Institutes of Health Stroke Scale score provided excellent discrimination $(86 \%$ specificity; $84 \%$ sensitivity) of type I outcomes. The receiver operating characteristic area under the curve for changes in MiniMental State Examination score $(\mathrm{n}=4707)$ was 0.75 for type I outcomes and 0.71 for type II outcomes. A 2-point worsening in Mini-Mental State Examination score provided only fair discrimination (73\% specificity; $62 \%$ sensitivity) of type II outcomes.

Conclusion: We used baseline controls and postoperative worsening in National Institutes of Health Stroke Scale and Mini-Mental State Examination scores to predict both serious adverse neurologic outcome and deterioration of intellectual function. Our findings provide the only reference for evaluating these tests that are used in cardiac surgical clinical trials. (J Thorac Cardiovasc Surg 2010;139:901-12)

\footnotetext{
From the Department of Anesthesiology, ${ }^{\text {a }}$ SUNY Upstate Medical University, Syracuse, NY; Ischemia Research and Education Foundation, ${ }^{\mathrm{b}}$ San Bruno, Calif; Cardiovascular Anesthesia Group, ${ }^{c}$ Kaiser-Permanente, and the University of California, San Francisco, Calif; Department of Anesthesiology, ${ }^{\mathrm{d}}$ University of Wisconsin Hospital and Clinics, Madison, Wis; Department of Anesthesia, ${ }^{\text {e Stan- }}$ ford University School of Medicine, Stanford, Calif; Department of Anaesthesia, ${ }^{f}$ The Liverpool Heart and Chest Hospital, Liverpool, United Kingdom; Department of Anesthesia, ${ }^{\mathrm{g}}$ Gottsegen György Hungarian Institute of Cardiology, Budapest, Hungry; Institute of Anesthesiology and Intensive Care, ${ }^{\text {h }}$ San Raffaele Hospital, and San Paolo University Hospital, Milan, Italy; and Department of Anesthesiology, Lehigh Valley Hospital and Health Network, Allentown, Pa.

* A complete list of the investigators and participating centers of the Ischemia Research and Education Foundation and the Multicenter Study of Perioperative Ischemia Research Group is at the end of this article.

Disclosures: None.

Received for publication Aug 19, 2008; revisions received June 2, 2009; accepted for publication July 22, 2009; available ahead of print Sept 11, 2009.

Address for reprints: Nancy A. Nussmeier, MD, SUNY Upstate Medical University (Syracuse, New York), c/o Editorial Office, Ischemia Research and Education Foundation, 1111 Bayhill Drive, Suite 480, San Bruno, CA 94066 (E-mail: nussmein@upstate.edu and diane@iref.org).

0022-5223/\$36.00

Copyright (c) 2010 by The American Association for Thoracic Surgery

doi:10.1016/j.jtcvs.2009.07.055
}

The National Institutes of Health Stroke Scale (NIHSS) is used to evaluate neurologic impairment. It was designed for use in clinical trials of interventions to ameliorate cerebral damage after acute stroke. ${ }^{1,2}$ This widely used scale has been recommended by the Stroke Council of the American Heart Association for serial assessment of patients with acute stroke. ${ }^{3}$ Although often used in studies of neurologic outcome after cardiac surgery, ${ }^{4-6}$ the NIHSS has never been specifically studied or validated as an appropriate instrument for use in this setting.

The Mini-Mental State Examination (MMSE) is one of the most widely used instruments for screening for dementia, ${ }^{7,8}$ particularly for Alzheimer's disease, and measuring cognitive change over time in older adults. It is also used as one of a battery of tests assessing neurocognitive outcome in studies of patients undergoing cardiac surgery. ${ }^{4,6,9}$ However, only 1 study (involving 100 consecutive patients) has prospectively evaluated the clinical utility of MMSE for screening in this setting. ${ }^{10}$ Although the investigators concluded that MMSE-based detection of cognitive dysfunction should warrant geriatric follow-up after hospital discharge, 


\section{Abbreviations and Acronyms \\ AUC = area under the curve \\ $\mathrm{CABG}=$ coronary artery bypass grafting \\ $\mathrm{CPB}=$ cardiopulmonary bypass \\ IREF = Ischemia Research and Education Foundation \\ MMSE $=$ Mini-Mental State Examination \\ NIHSS $=$ National Institutes of Health Stroke Scale \\ $\mathrm{ROC}=$ receiver operating characteristic}

their study was too small to correlate changes on the MMSE with overt neurologic sequelae.

We have previously described the classification of new overt adverse neurologic sequelae after cardiac surgery into 2 general categories: type I (death due to stroke or hypoxic encephalopathy, nonfatal stroke, transient ischemic attack, or stupor or coma at the time of discharge) and type II (new deterioration in intellectual function, confusion, agitation, disorientation, memory deficit, or seizure without evidence of focal injury). ${ }^{11}$ Our prior study, however, used neither the NIHSS nor the MMSE to assess neurologic outcome. ${ }^{11}$ In the current study, we wanted to determine whether postoperative changes from baseline in NIHSS scores, MMSE scores, or both would be sensitive or specific to either type I or type II neurologic outcomes. We hypothesized that the NIHSS would be sensitive and specific for detecting overt new postoperative type I outcomes but less so for type II outcomes. However, the MMSE, as a single screening test, might not be sensitive or specific for either type I or type II neurologic outcomes. To test these hypotheses, we analyzed preoperative and postoperative NIHSS and MMSE scores that were gathered prospectively in a large, international, multicenter study.

\section{MATERIALS AND METHODS Study Design and Patients}

The Multicenter Study of Perioperative Ischemia Epidemiology II, funded by the Ischemia Research and Education Foundation (IREF), is a retrospective analysis of prospectively collected data in patients undergoing coronary artery bypass grafting (CABG) surgery at 72 hospitals in 17 countries between November 1996 and June 2000. A systematic sampling scheme $^{12}$ was used to select up to 100 patients at each site, aged 18 years or older, undergoing CABG surgery with or without valve repair or replacement while on cardiopulmonary bypass (CPB). All participating sites in the study were required to submit their institutional review board approval to IREF to obtain authorization to join the study. Written informed consent was obtained from each patient before enrollment could ensue.

Of the 5436 patients enrolled (Figure 1), 371 were excluded from the data analyses because of patient withdrawal $(n=32)$, death before surgery $(n=2)$, canceled or rescheduled surgery $(n=97)$, alteration of surgical schedule $(n=132)$, enrollment in another study $(n=11)$, or incomplete data collection ( $\mathrm{n}=97$; see Appendix 1). Also excluded were 358 patients who underwent a carotid procedure concurrently with CABG $(\mathrm{n}=56)$, patients who underwent emergency CABG because of life-threatening conditions $(\mathrm{n}=12)$, patients who had severe hematologic disorders $(\mathrm{n}=12)$, patients who had religious beliefs precluding transfusion $(n=5)$, patients who had a previous deforming injury such as amputation $(\mathrm{n}=17)$, and patients who had a known history of intravenous drug use or alcohol abuse $(\mathrm{n}=271)$.

\section{Test Administration}

Standardized NIHSS certification procedures were followed. ${ }^{13,14}$ At each site, all personnel planning to administer the NIHSS received training and were required to pass a certification test. A standardized NIHSS training videotape provided demonstration cases and detailed instructions regarding test procedures and scoring of each scale item. Individuals seeking certification then viewed a separate videotape that required completion of the NIHSS for each of 5 standardized patients. Answers were submitted to IREF, an organization accredited for certification. The individual seeking certification was required to have a test score of at least 100 correct answers of a possible total of 105 . Each site was notified of the certification status of its research personnel. Certification test data for each test giver from each participating site were stored in the IREF database.

Administration of the MMSE does not require certification. However, all clinical personnel conducting this examination attended a training session. Furthermore, each test giver received printed instructions in his/her native language for each question on the examination.

\section{Data Collection and Management}

Data were collected from each patient by independent investigators throughout each patient's hospitalization. The collected data included demographic, historical, clinical, laboratory, electrocardiographic, surgical, and other intraoperative information, and data regarding adverse outcomes and resource use. All data fields for each patient were queried for completeness and accuracy, with all changes documented before database closure. All outcomes were defined in advance and determined by independent investigators blinded to the study question.

The primary outcome variables were type I or II overt neurologic sequelae. Type I outcomes were defined as any of the following: death due to stroke or hypoxic encephalopathy, nonfatal stroke, transient ischemic attack, or stupor or coma at the time of discharge. Type II outcomes were defined, according to a "2-day, 2-item" rule, as the presence of 2 or more indications of intellectual dysfunction (eg, confusion, disorientation, agitation, memory deficits, or seizures) on 2 or more days, not necessarily consecutive. All final type I and type II outcomes were determined by committee. The primary predictor variable for both type I and type II outcomes was postoperative worsening in NIHSS or MMSE score from the preoperative baseline score. Receiver operating characteristic (ROC) curves were created and used to determine the predictive value of worsening in NIHSS or MMSE scores with regard to type I and II outcomes. The sensitivity, specificity, positive predictive value, negative predictive value, and likelihood ratio of the NIHSS or MMSE score are reported.

The NIHSS and MMSE examinations were routinely performed per the study protocol on postoperative day 3,4 , or 5 by investigators certified in administration of these tests. On the NIHSS, a perfect score is zero, each point on the neurologic deficit scale is assigned a whole number, the maximum number of possible points is 42 , and higher scores indicate worsening neurologic function. On the MMSE, a perfect score is 30 , each wrong answer is assigned a point value (ie, a whole number), and lower scores indicate worsening neurocognitive dysfunction. To establish the clinical utility of NIHSS and MMSE scores in prediction of postoperative type I or II outcomes in cardiac surgical patients, analyses for ROC curve, sensitivity, and specificity were performed for worsening of score (postoperative score minus preoperative score).

For missing data regarding potential predictors, a combined variables approach was used. For missing NIHSS or MMSE scores, most study patients were examined but had missing records for a few individual questions; thus, imputation was performed by dividing the patients into 4 groups: female aged $\leq 70$ years, male aged $\leq 70$ years, female aged $>70$ years, and 


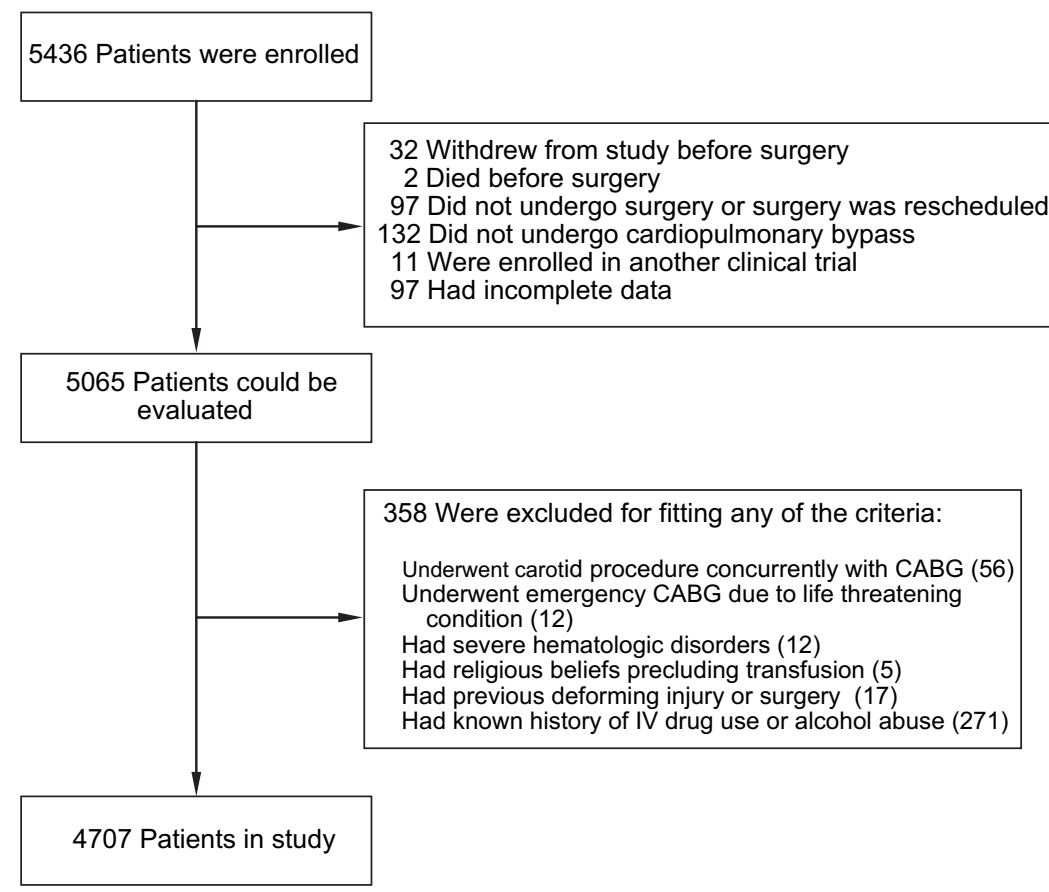

FIGURE 1. Consolidated Standards of Reporting Trials diagram of patient enrollment. $C A B G$, Coronary artery bypass grafting.

male aged $>70$ years. The mean score was calculated for each question on the NIHSS or MMSE, stratified by age and gender, separately for the preoperative and postoperative periods. Missing data for the individual question for each patient were imputed using the mean score of the question and then sum scores for all questions.

Baseline characteristics, which included demographic, historical, preoperative and intraoperative factors, and univariate outcomes, were compared among patients without type I or II outcome, patients with type I outcome, and patients with type II outcome (see Appendix 2). The Kruskal-Wallis test and chi-square test were used for group comparisons among continuous and categorical variables, respectively.

To assess the independent effects of predictors on "subnormal" (greater than zero) preoperative or postoperative NIHSS score, a Zero-inflated Negative binomial model with a random effect of country (the NLMIXED procedure in SAS software; SAS Institute, Inc, Cary, NC) was used. This will account for the excess of zeros in NIHSS scores. ${ }^{15}$ A Logit model with binomial assumption was used to account for the probability of a zero-NIHSS score. Then a Negative binomial regression was performed to model the count outcome in the not-always-zero group. The preoperative subnormal NIHSS score model was adjusted for baseline characteristics and countries. The additional adjustments for preoperative NIHSS score and intraoperative factors were added in the postoperative adverse NIHSS score model.

To evaluate MMSE scores, we defined a "subnormal" MMSE score according to the score distribution and the analysis of ROC curve, sensitivity, and specificity of preoperative or postoperative MMSE scores with regard to type I or II outcomes and literature review. ${ }^{16,17}$ Both preoperative and postoperative MMSE scores were categorized as "high score" (normal) $=27$ 30 , "moderate score" $=24-26$, and "low score" $($ subnormal $)=0-23$. Generalized estimating equations regression models for ordinal outcomes (the GENMOD procedure) were performed to assess the associations between subnormal MMSE scores and predictors and account for the clustering of patients within countries. The preoperative subnormal MMSE score model was adjusted for baseline characteristics and countries. Additional adjustments for preoperative MMSE score and intraoperative factors were added in the postoperative subnormal MMSE score model.
We developed a series of multivariable models to assess the independent effects of predictors on the type I or II outcome and stroke (fatal or nonfatal). The models were adjusted for baseline characteristics and intraoperative factors. Generalized estimating equations models (the GENMOD procedure) were used to account for the clustering of patients within countries. All analyses were performed with SAS software, version 8.12.

\section{RESULTS}

Of the 4707 study patients (Table 1), 2.9\% (135/4707) had new postoperative type I outcomes, and 6.4\% (301/ 4707) had type II outcomes. Resource use, measured by length of postoperative intensive care unit and hospital stays and discharge to a facility other than home, was significantly greater among patients with worse outcomes (Table 1). The median time of postoperative NIHSS examination was on the fourth postoperative day. The area under the ROC curve (AUC) for changes in NIHSS score was 0.89 for type I outcomes and 0.66 for type II outcomes (Table 2). There was little change in the AUC when correction factors were applied for age and higher education (college or postgraduate education) (Table 2).

A 1-point worsening in NIHSS score provided $86 \%$ specificity and $84 \%$ sensitivity for type I outcomes, and a $2-$ point worsening in the NIHSS score provided $94 \%$ specificity and $71 \%$ sensitivity (Table 3). For type II outcomes, a 1-point worsening in the NIHSS score provided $88 \%$ specificity and $44 \%$ sensitivity, and a 2 -point worsening in the score provided $95 \%$ specificity but only $33 \%$ sensitivity (Table 3 ).

The median time for performance of MMSE examination was postoperative day 4 . The AUC for changes in MMSE 
TABLE 1. Characteristics of study patients $(n=4707) *$

\begin{tabular}{|c|c|c|c|c|c|}
\hline Characteristic & $\begin{array}{c}\text { All patients } \\
(n=4707) \\
\end{array}$ & $\begin{array}{c}\text { Patients without } \\
\text { type I or II outcome } \\
(n=4271) \\
\end{array}$ & $\begin{array}{r}\text { Patients with type } I \\
\text { outcome }(n=135) \\
\end{array}$ & $\begin{array}{c}\text { Patients with type II } \\
\text { outcome }(\mathrm{n}=301)\end{array}$ & $P$ value \\
\hline Age (y) & & & & & $<.001$ \\
\hline Median & 64.9 & 64.1 & 69.5 & 71.3 & \\
\hline Interquartile range & $57.5-71.6$ & $57.0-71.0$ & $63.9-74.3$ & $65.2-75.9$ & \\
\hline $\begin{array}{l}\left.\text { Obesity (BMI }>28 \mathrm{~kg} / \mathrm{m}^{2}\right), \\
\text { no. }(\%)\end{array}$ & $1861(39.5)$ & 1690 (39.6) & $55(40.7)$ & $116(38.5)$ & .90 \\
\hline Diabetes, no. $(\%)$ & $1416(30.1)$ & $1242(29.1)$ & $56(41.5)$ & $118(39.2)$ & $<.001$ \\
\hline $\begin{array}{l}\text { College or postgraduate } \\
\text { education, no. }(\%)\end{array}$ & $850(18.1)$ & $781(18.3)$ & $26(19.3)$ & $43(14.3)$ & .20 \\
\hline $\begin{array}{l}\text { African American, } \\
\text { American Indian, or } \\
\text { Hispanic ethnicity }\end{array}$ & $355(7.5)$ & $318(7.4)$ & $11(8.1)$ & $26(8.6)$ & .72 \\
\hline \multicolumn{6}{|l|}{$\begin{array}{l}\text { Medical history or } \\
\text { preoperative factor, no. }(\%)\end{array}$} \\
\hline Congestive heart failure & $282(6.0)$ & $224(5.2)$ & $18(13.3)$ & $40(13.3)$ & $<.001$ \\
\hline Myocardial infarction & $2489(52.9)$ & $2239(52.4)$ & $74(54.8)$ & $176(58.5)$ & .11 \\
\hline Unstable angina & $2638(56.0)$ & $2357(55.2)$ & $88(65.2)$ & $193(64.1)$ & .001 \\
\hline $\begin{array}{l}\text { Atrial fibrillation or } \\
\text { atrial flutter }\end{array}$ & $392(8.3)$ & $331(7.7)$ & $21(15.6)$ & $40(13.3)$ & $<.001$ \\
\hline Dysrhythmia & $891(18.9)$ & $777(18.2)$ & $35(25.9)$ & $79(26.2)$ & $<.001$ \\
\hline Neurologic dysfunction & $512(10.9)$ & $420(9.8)$ & $36(26.7)$ & $56(18.7)$ & $<.001$ \\
\hline Mental dysfunction & $717(15.2)$ & $619(14.5)$ & $31(23.0)$ & $67(22.3)$ & $<.001$ \\
\hline Hypertension & $3284(69.8)$ & $2933(68.7)$ & $108(80.0)$ & $243(80.7)$ & $<.001$ \\
\hline Peripheral vascular disease & $777(16.5)$ & $673(15.8)$ & $31(23.0)$ & $73(24.3)$ & $<.001$ \\
\hline Carotid vascular disease & $714(15.2)$ & $601(14.1)$ & $38(28.1)$ & $75(24.9)$ & $<.001$ \\
\hline Aortic vascular disease & $707(15.0)$ & $610(14.3)$ & $28(20.7)$ & $69(22.9)$ & $<.001$ \\
\hline Renal disease & $136(2.9)$ & $106(2.5)$ & $7(5.2)$ & $23(7.6)$ & $<.001$ \\
\hline Valve disease & $910(19.3)$ & $758(17.8)$ & $42(31.1)$ & $110(36.5)$ & $<.001$ \\
\hline Pulmonary disease & $664(14.1)$ & $578(13.5)$ & $30(22.2)$ & $56(18.6)$ & .001 \\
\hline $\begin{array}{l}\text { Preoperative pulse } \\
\text { pressure, } \mathrm{mm} \mathrm{Hg}\end{array}$ & & & & & $<.001$ \\
\hline Median & 57.0 & 56.5 & 62.5 & 60.0 & \\
\hline Interquartile range & $48.0-67.5$ & $48.0-67.0$ & $52.0-76.0$ & $48.0-71.0$ & \\
\hline $\begin{array}{l}\text { Preoperative insertion } \\
\text { of IABP }\end{array}$ & $34(0.7)$ & $31(0.7)$ & $0(0.0)$ & $3(1.0)$ & .52 \\
\hline Redo operation & $311(6.6)$ & $267(6.3)$ & $15(11.1)$ & $29(9.6)$ & .008 \\
\hline \multicolumn{6}{|l|}{ Surgical factor, no. $(\%)$} \\
\hline $\begin{array}{l}\text { Concomitant current } \\
\text { procedure }\end{array}$ & $655(13.9)$ & $541(12.7)$ & $34(25.2)$ & $80(26.6)$ & $<.001$ \\
\hline $\begin{array}{l}\text { Concurrent } \mathrm{CABG} \text { and } \\
\text { valve (aortic/mitral) surgery }\end{array}$ & $508(10.8)$ & $419(9.8)$ & $26(19.3)$ & $63(20.9)$ & $<.001$ \\
\hline $\begin{array}{c}\text { Bypass of }>3 \text { proximal } \\
\text { aortic anastomoses }\end{array}$ & $191(4.1)$ & $160(3.7)$ & $13(9.6)$ & $18(6.0)$ & $<.001$ \\
\hline $\begin{array}{c}\text { Intraoperative use of } \\
\text { antifibrinolytics } \dagger\end{array}$ & $2751(67.6)$ & $2477(67.1)$ & $84(70.6)$ & $190(74.2)$ & .05 \\
\hline Intraoperative hypertension $\ddagger$ & $1678(36.4)$ & $1478(35.4)$ & $62(46.6)$ & $138(46.5)$ & $<.001$ \\
\hline $\begin{array}{l}\text { Intraoperative atrial } \\
\text { fibrillation or atrial flutter }\end{array}$ & $345(7.3)$ & $296(6.9)$ & $18(13.3)$ & $31(10.3)$ & .002 \\
\hline $\begin{array}{l}\text { Intraoperative homologous } \\
\text { red blood cell transfusion }\end{array}$ & $1767(37.6)$ & $1525(35.7)$ & $61(45.2)$ & $181(60.1)$ & $<.001$ \\
\hline $\begin{array}{l}\text { Intraoperative homologous } \\
\text { fresh-frozen plasma } \\
\text { transfusion }\end{array}$ & $487(10.4)$ & $395(9.3)$ & $27(20.0)$ & 65 (21.6) & $<.001$ \\
\hline
\end{tabular}


TABLE 1. Continued

\begin{tabular}{|c|c|c|c|c|c|}
\hline Characteristic & $\begin{array}{l}\text { All patients } \\
(\mathrm{n}=4707)\end{array}$ & $\begin{array}{l}\text { Patients without } \\
\text { type I or II outcome } \\
(n=4271)\end{array}$ & $\begin{array}{c}\text { Patients with type I } \\
\text { outcome }(n=135)\end{array}$ & $\begin{array}{l}\text { Patients with type II } \\
\text { outcome }(n=301)\end{array}$ & $P$ value \\
\hline $\begin{array}{l}\text { Intraoperative homologous } \\
\text { platelets transfusion }\end{array}$ & $424(9.0)$ & $346(8.1)$ & $19(14.1)$ & $59(19.6)$ & $<.001$ \\
\hline Return to $\mathrm{CPB}$ & $210(4.5)$ & $175(4.1)$ & $13(9.6)$ & $22(7.3)$ & $<.001$ \\
\hline Duration of CPB (min) & & & & & $<.001$ \\
\hline Median & 96.0 & 94.0 & 107.0 & 109.0 & \\
\hline Interquartile range & $74.0-123.0$ & $73.0-121.0$ & $86.0-136.0$ & $82.0-142.0$ & \\
\hline \multicolumn{6}{|l|}{ Postoperative factor, no. ( $\%)$} \\
\hline Postoperative renal composite $\S$ & $226(4.8)$ & $145(3.4)$ & $30(22.2)$ & $51(16.9)$ & $<.001$ \\
\hline Length of ICU stay (d) & & & & & $<.001$ \\
\hline Median & 1.3 & 1.2 & 3.9 & 4.2 & \\
\hline Interquartile range & $0.9-2.8$ & $0.9-2.3$ & $1.5-9.3$ & $1.8-8.0$ & \\
\hline Length of hospital stay (d) & & & & & $<.001$ \\
\hline Median & 8.0 & 8.0 & 13.0 & 11.0 & \\
\hline Interquartile range & $6.0-11.0$ & $6.0-10.0$ & $10.0-21.0$ & $8.0-18.0$ & \\
\hline $\begin{array}{l}\text { Discharge to facility } \\
\text { other than home }\end{array}$ & $1533(33.6)$ & $1321(31.7)$ & $64(56.1)$ & $148(53.4)$ & $<.001$ \\
\hline Patient died in hospital & $151(3.2)$ & $106(2.5)$ & $21(15.6)$ & $24(8.0)$ & $<.001$ \\
\hline \multicolumn{6}{|c|}{ 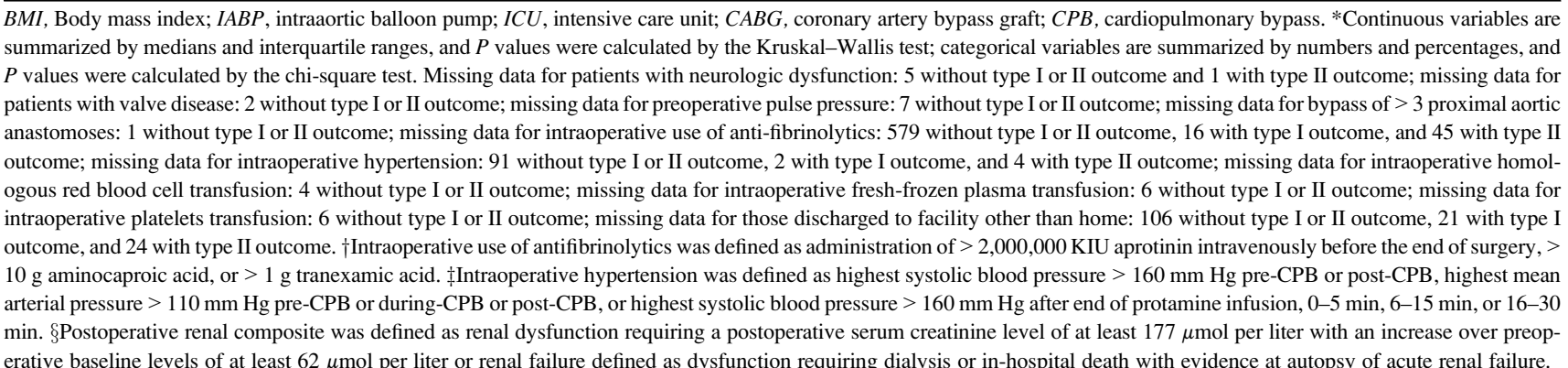 } \\
\hline
\end{tabular}

score was 0.75 for type I outcomes and 0.71 for type II outcomes (Table 2). There was little change in the AUC when correction factors were applied for age and higher education (college or postgraduate education) (Table 2). A 2-point worsening in MMSE score provided only $73 \%$ specificity and $63 \%$ sensitivity for type I outcomes (Table 3). For type II outcomes, a 2-point worsening in the MMSE score provided $73 \%$ specificity and $62 \%$ sensitivity (Table 3 ).

For the NIHSS, $37 \%$ of patients were tested on postoperative day $3,30 \%$ of patients were tested on postoperative day 4 , and $22 \%$ of patients were tested on postoperative day 5 . For the MMSE, $38 \%$ of patients were tested on postoperative day $3,30 \%$ of patients were tested on postoperative day 4 , and $22 \%$ of patients were tested on postoperative day 5 . There were small, statistically significant but clinically unimportant differences in the NIHSS and MMSE results according to the postoperative date of testing (on postoperative day 3,4 , or 5 ). These differences are reported in Appendices 3 and 4.

We also examined the predictive value of both the NIHSS and MMSE if only nonfatal or fatal stroke outcomes are considered. The sensitivity and specificity of both the NIHSS and MMSE were better for stroke only (Table 4). The
AUC for changes in NIHSS score was 0.95, and the AUC for changes in MMSE score was 0.77.

Multivariate analysis determined that the common predictors of worsening postoperative NIHSS and MMSE scores included age increment, performance on the preoperative NIHSS and MMSE, a history of neurologic dysfunction,

TABLE 2. Receiver operating characteristic for worsening in National Institutes of Health Stroke Scale or Mini-Mental State Examination scores predicting type I or II outcomes*

\begin{tabular}{cccc}
\hline Test & Adjustment & $\begin{array}{c}\text { ROC for } \\
\text { prediction } \\
\text { of type I outcome }\end{array}$ & $\begin{array}{c}\text { ROC for } \\
\text { prediction of } \\
\text { type II outcome }\end{array}$ \\
\hline NIHSS & Without adjustment & 0.89 & 0.66 \\
NIHSS & $\begin{array}{c}\text { Adjustment for age } \dagger \\
\text { and education } \ddagger\end{array}$ & 0.89 & 0.73 \\
MMSE & Without adjustment & 0.75 & 0.71 \\
MMSE & $\begin{array}{c}\text { Adjustment for age } \dagger \\
\text { and education } \ddagger\end{array}$ & 0.75 & 0.77 \\
\hline
\end{tabular}

ROC, Receiver operating characteristic; NIHSS, National Institutes of Health Stroke Scale; MMSE, Mini-Mental State Examination. *Type I, event(s) before postoperative NIHSS test or MMSE test, or type I-neurologic death. $\nmid$ Age, age $>60 \mathrm{y}$ and per $5 \mathrm{y}$ thereof, and $>80 \mathrm{y}$. ‡Education, with higher education (college or postgraduate education) vs without higher education. 
TABLE 3. Sensitivity, specificity, positive predictive value, negative predictive value, and likelihood ratio of National Institutes of Health Stroke Scale or Mini-Mental State Examination score for type I or II outcomes

\begin{tabular}{|c|c|c|c|c|c|c|c|c|}
\hline $\begin{array}{l}\text { CNS type I or } \\
\text { II outcome }\end{array}$ & $\begin{array}{l}\text { Worsening in } \\
\text { NIHSS or } \\
\text { MMSE score }\end{array}$ & $\begin{array}{c}\text { ROC } \\
\text { curves }\end{array}$ & Specificity & Sensitivity & $\begin{array}{c}\text { Positive } \\
\text { predictive value } \\
\end{array}$ & $\begin{array}{c}\text { Negative } \\
\text { predictive value }\end{array}$ & $\begin{array}{c}\text { Likelihood } \\
\text { ratio-positive } \\
\text { test result }\end{array}$ & $\begin{array}{c}\text { Likelihood } \\
\text { ratio-negative } \\
\text { test result } \\
\end{array}$ \\
\hline Type I* & NIHSS change $\geq 1$ & 0.85 & 0.86 & 0.84 & 0.10 & 1.00 & 6.20 & 0.19 \\
\hline Type I* & NIHSS change $\geq 2$ & 0.83 & 0.94 & 0.71 & 0.19 & 0.99 & 12.79 & 0.30 \\
\hline Type I* & NIHSS change $\geq 3$ & 0.75 & 0.97 & 0.54 & 0.25 & 0.99 & 18.22 & 0.48 \\
\hline Type I* & NIHSS change $\geq 4$ & 0.74 & 0.98 & 0.49 & 0.35 & 0.99 & 28.85 & 0.52 \\
\hline Type II & NIHSS change $\geq 1$ & 0.66 & 0.88 & 0.44 & 0.19 & 0.96 & 3.66 & 0.63 \\
\hline Type II & NIHSS change $\geq 2$ & 0.64 & 0.95 & 0.33 & 0.31 & 0.96 & 6.95 & 0.70 \\
\hline Type II & NIHSS change $\geq 3$ & 0.61 & 0.98 & 0.24 & 0.38 & 0.95 & 9.84 & 0.78 \\
\hline Type II & NIHSS change $\geq 4$ & 0.57 & 0.98 & 0.16 & 0.38 & 0.95 & 9.60 & 0.86 \\
\hline Type I* & MMSE change $\leq-1$ & 0.66 & 0.54 & 0.79 & 0.03 & 0.99 & 1.71 & 0.39 \\
\hline Type I* & MMSE change $\leq-2$ & 0.68 & 0.73 & 0.63 & 0.05 & 0.99 & 2.29 & 0.51 \\
\hline Type I* & MMSE change $\leq-3$ & 0.69 & 0.84 & 0.54 & 0.06 & 0.99 & 3.30 & 0.55 \\
\hline Type I* & MMSE change $\leq-4$ & 0.69 & 0.90 & 0.48 & 0.09 & 0.99 & 4.84 & 0.57 \\
\hline Type II & MMSE change $\leq-1$ & 0.65 & 0.54 & 0.75 & 0.10 & 0.97 & 1.63 & 0.46 \\
\hline Type II & MMSE change $\leq-2$ & 0.68 & 0.73 & 0.62 & 0.14 & 0.97 & 2.35 & 0.51 \\
\hline Type II & MMSE change $\leq-3$ & 0.66 & 0.85 & 0.48 & 0.18 & 0.96 & 3.18 & 0.62 \\
\hline Type II & MMSE change $\leq-4$ & 0.65 & 0.91 & 0.39 & 0.23 & 0.96 & 4.48 & 0.67 \\
\hline
\end{tabular}

$C N S$, Central nervous system; NIHSS, National Institutes of Health Stroke Scale; ROC, receiver operating characteristic; MMSE, Mini-Mental State Examination. *Type I, event(s) before postoperative NIHSS test or MMSE test, or type I-neurologic death.

diabetes, intraoperative transfusion of red blood cells, and duration of CPB (Table 5). For the NIHSS, additional predictors of worsening score included congestive heart failure, hypertension, and pulmonary disease. For the MMSE, additional predictors of worsening score included female gender, a history of renal disease, valvular heart disease, and intraoperative hypertension, whereas higher education was "protective."

In regard to the predictors of type I and type II outcomes, multivariate analysis determined that the common predictors of these overt adverse events were age increment, diabetes, and intraoperative fresh-frozen plasma transfusion (Table 5). For type I outcomes, additional predictors included neurologic dysfunction, congestive heart failure, high pulse pressure, and more than 3 proximal aortic anastomoses. For type II outcomes, additional predictors included a history of myo- cardial infarction, renal disease, or valvular heart disease, as well as intraoperative hypertension and intraoperative transfusion of red blood cells. If only nonfatal or fatal stroke outcomes are considered, multivariate analysis confirmed that preoperative predictors were neurologic dysfunction, carotid disease, pulmonary disease, high pulse pressure, and redo surgery (Table 6).

The median preoperative NIHSS score was zero (zero being a perfect score) with an interquartile range of 0 to 0 ; $85 \%$ of study patients had a preoperative NIHSS score of zero. The median preoperative MMSE score was 29 (30 being a perfect score) with an interquartile range of 27 to 30. Common preoperative predictors of "poor" preoperative NIHSS (Figure 2, $A$ ) or MMSE (Figure 2, $B$ ) scores were age increment, congestive heart failure, and valvular heart disease. For the NIHSS, additional preoperative

TABLE 4. Sensitivity, specificity, positive predictive value, negative predictive value, and likelihood ratio of National Institutes of Health Stroke Scale or Mini-Mental State Examination Score for nonfatal or fatal stroke ( $n=92$ patients)

\begin{tabular}{|c|c|c|c|c|c|c|c|}
\hline $\begin{array}{c}\text { Worsening in NIHSS } \\
\text { or MMSE score }\end{array}$ & $\begin{array}{l}\text { ROC* } \\
\text { curves }\end{array}$ & Specificity & Sensitivity & $\begin{array}{c}\text { Positive } \\
\text { predictive value }\end{array}$ & $\begin{array}{c}\text { Negative } \\
\text { predictive value }\end{array}$ & $\begin{array}{l}\text { Likelihood ratio- } \\
\text { positive test result }\end{array}$ & $\begin{array}{l}\text { Likelihood ratio- } \\
\text { negative test result }\end{array}$ \\
\hline NIHSS change $\geq 1$ & 0.91 & 0.86 & 0.95 & 0.09 & 1.00 & 6.96 & 0.06 \\
\hline NIHSS change $\geq 2$ & 0.88 & 0.94 & 0.82 & 0.17 & 1.00 & 14.38 & 0.19 \\
\hline NIHSS change $\geq 3$ & 0.80 & 0.97 & 0.63 & 0.23 & 0.99 & 20.49 & 0.38 \\
\hline NIHSS change $\geq 4$ & 0.78 & 0.98 & 0.58 & 0.32 & 0.99 & 32.44 & 0.43 \\
\hline MMSE change $\leq-1$ & 0.68 & 0.54 & 0.83 & 0.03 & 0.99 & 1.79 & 0.32 \\
\hline MMSE change $\leq-2$ & 0.70 & 0.73 & 0.67 & 0.04 & 0.99 & 2.44 & 0.45 \\
\hline MMSE change $\leq-3$ & 0.70 & 0.84 & 0.57 & 0.05 & 0.99 & 3.47 & 0.51 \\
\hline MMSE change $\leq-4$ & 0.71 & 0.90 & 0.53 & 0.08 & 0.99 & 5.20 & 0.52 \\
\hline
\end{tabular}

NIHSS, National Institutes of Health Stroke Scale; MMSE, Mini-Mental State Examination; ROC, receiver operating characteristic. *Nonfatal stroke, stroke before postoperative NIHSS test or MMSE test. 
TABLE 5. Model-adjusted ratios for type I or type II cerebral outcome, or worsening National Institutes of Health Stroke Scale or Mini-Mental State Examination scores associated with selected factors*

\begin{tabular}{|c|c|c|c|c|c|}
\hline \multirow[b]{2}{*}{ Predictor } & \multirow[t]{2}{*}{$\begin{array}{c}\text { Type I CNS } \\
\text { outcome }\end{array}$} & \multirow[t]{2}{*}{$\begin{array}{c}\text { Type II } \\
\text { CNS outcome }\end{array}$} & \multicolumn{2}{|c|}{$\begin{array}{l}\text { Postoperative } \\
\text { NIHSS Score } \dagger\end{array}$} & \multirow[t]{2}{*}{$\begin{array}{l}\text { Postoperative } \\
\text { MMSE score } \neq\end{array}$} \\
\hline & & & Logit portion & $\begin{array}{c}\text { Negative } \\
\text { binomial portion }\end{array}$ & \\
\hline & OR $(95 \%$ CI $)$ & OR $(95 \%$ CI $)$ & OR $(95 \%$ CI $)$ & $\mathbf{R R}(\mathbf{9 5} \% \mathbf{C I})$ & OR $(95 \%$ CI $)$ \\
\hline $\begin{array}{l}\text { Age }>60 y \text { and per } 5 y \text { thereof } \\
\text { and }>80 y\end{array}$ & $1.27(1.14-1.41) \Phi$ & $1.43(1.34-1.53) \Phi$ & $0.84(0.76-0.92) \rrbracket$ & $1.17(1.11-1.24) \Phi$ & $1.29(1.21-1.38)$ ฯ \\
\hline College or postgraduate education & & & & & $0.68(0.55-0.83)$ ฯ \\
\hline Women & & & & & $1.24(1.08-1.43)$ \\
\hline \multicolumn{6}{|l|}{ Medical history/preoperative factor } \\
\hline Preoperative NIHSS/MMSE score & & & & $1.20(1.13-1.27) \Phi$ & $1.51(1.45-1.58)$ ฯ \\
\hline Neurologic dysfunction & $2.38(1.58-3.58) \uparrow$ & & & $1.54(1.25-1.89) \Phi$ & $1.32(1.10-1.59)$ \\
\hline Congestive heart failure & $2.03(1.00-4.10)$ & & & $1.33(1.02-1.74)$ & \\
\hline Myocardial infarction & & $1.42(1.12-1.78)$ & & & \\
\hline Hypertension & & & & $1.43(1.17-1.76) \Phi$ & \\
\hline $\begin{array}{l}\text { Pulse pressure (per } 10 \mathrm{~mm} \mathrm{Hg} \\
\text { increment) } \S\end{array}$ & $1.17(1.05-1.30)$ & & & & \\
\hline Pulmonary disease & & & & $1.52(1.23-1.88) \Phi$ & \\
\hline Diabetes & $1.54(1.17-2.04)$ & $1.48(1.18-1.85) \Upsilon$ & & $1.42(1.18-1.69) \Phi$ & $1.25(1.14-1.38)$ ฯ \\
\hline Renal disease & & $2.20(1.12-4.32)$ & & & $1.74(1.31-2.32)$ ฯ \\
\hline \multicolumn{6}{|l|}{ Intraoperative factors } \\
\hline$>3$ proximal aortic anastomoses & $2.43(1.41-4.17)$ & & & & \\
\hline Valvular heart disease & & $1.77(1.40-2.24) \Phi$ & & & $1.29(1.15-1.44) \Phi$ \\
\hline Intraoperative hypertension $\|$ & & $1.36(1.17-1.59) \Phi$ & & & $1.17(1.03-1.32)$ \\
\hline $\begin{array}{l}\text { Intraoperative homologous RBC } \\
\text { transfusion }\end{array}$ & & $1.48(1.14-1.92)$ & & $1.36(1.12-1.64)$ & $1.34(1.14-1.56)$ ฯ \\
\hline $\begin{array}{l}\text { Intraoperative homologous FFP } \\
\text { transfusion }\end{array}$ & $1.99(1.54-2.58) \Phi$ & $1.54(1.14-2.09)$ & & & \\
\hline CPB time per 30-min increment & & & $0.87(0.78-0.97)$ & $1.06(1.001-1.13)$ & $1.15(1.09-1.22) \Phi$ \\
\hline $\begin{array}{l}C N S \text {, Central nervous system; NIHSS, Natio } \\
R B C \text {, red blood cells; } F F P \text {, fresh-frozen plas } \\
\text { or intraoperative factors, and countries. } \text { Zero } \\
\text { used to model the probability of zero-NIHS } \\
\text { operative NIHSS score was calculated per } 1 \\
\text { MMSE score and predictors; postoperative } \\
\text { as per } 1 \text { unit decrease. } \S \text { Pulse pressure per } 10 \\
\mathrm{~mm} \text { Hg pre-CPB or post-CPB, highest mear } \\
\text { protamine infusion } 0-5 \mathrm{~min}, 6-15 \mathrm{~min} \text {, or } 1\end{array}$ & $\begin{array}{l}1 \text { Institutes of Health Str } \\
; C P B \text {, cardiopulmonary } \\
\text { fflated Negative binomia } \\
\text { core, and then a Negativ } \\
\text { it increase. } \ddagger \text { Generalized } \\
\text { ISE score is categorized } \\
\text { Im Hg increment above a } \\
\text { rterial pressure }>110 \mathrm{~m} \\
-30 \text { min. } P P \text { value }<.00\end{array}$ & $\begin{array}{l}\text { Scale; MMSE, Mini-M } \\
\text { ass. *Models were adj } \\
\text { del is used to assess the } \\
\text { nomial model is perfor } \\
\text { nating equations mode } \\
\text { gh (27-30), moderate } \\
\text { shold of } 40 \mathrm{~mm} \mathrm{Hg.} \| 1 \\
\text { Ig pre-CPB or during-C }\end{array}$ & $\begin{array}{l}\text { al State Examination; } \\
\text { d for the patients' deme } \\
\text { ociation between posto } \\
\text { d to model count outco } \\
\text { ordinal outcome is use } \\
-26) \text {, and low }(<24) \text {. O } \\
\text { aoperative hypertension } \\
\text { or post-CPB, or highe }\end{array}$ & $\begin{array}{l}\text { odds ratio; } C I \text {, confiden } \\
\text { phic characteristics, me } \\
\text { tive NIHSS score and p } \\
\text { in the not-always-zero } \\
\text { assess the association o } \\
\text { atio for preoperative Ml } \\
\text { s defined as highest syst } \\
\text { ystolic blood pressure }\end{array}$ & $\begin{array}{l}\text { aterval; } R R \text {, rate ratio; } \\
1 \text { history, preoperative } \\
\text { ctors; a Logit model is } \\
\text { up. Rate ratio for pre- } \\
\text { rsening postoperative } \\
\text { Escore was calculated } \\
\text { blood pressure }>160 \\
0 \mathrm{~mm} \mathrm{Hg} \text { after end of }\end{array}$ \\
\hline
\end{tabular}

predictors of a "poor" score were a history of neurologic dysfunction and renal disease. For the MMSE, additional preoperative predictors of a "poor" score were a history of mental dysfunction, unstable angina, and peripheral vascular disease, whereas higher education was "protective.,"

\section{DISCUSSION}

We have reported preoperative and postoperative NIHSS and MMSE scores in a large international sample of patients undergoing cardiac surgery with CPB and the relationship of these scores to type I and II neurologic sequelae of surgery. Our findings provide the only reference for evaluating changes in NIHSS or MMSE scores from baseline, when these tests are used in clinical trials designed to diagnose, mitigate, or treat adverse neurologic outcome after cardiac surgery. We also defined predictors for type I and II adverse neurologic outcomes and compared these with predictors for postoperative worsening in NIHSS and MMSE scores. Finally, we explored patient characteristics that predict preoperative subnormal NIHSS and MMSE scores.

The NIHSS is a reliable $\mathrm{e}^{2,13,14}$ and validated ${ }^{1,18,19}$ measure of stroke-related neurologic impairment. It measures several aspects of brain function, including consciousness, vision, sensation, movement, speech, and language, with a maximal score of 42 representing the most severe and devastating stroke. A normal score is zero (no stroke), a score of 1 to 4 indicates a minor stroke, a score of 5 to 15 indicates a moderate stroke, a score of 15 to 20 indicates a moderate-to-severe stroke, and a score of 21 to 42 indicates a severe stroke. ${ }^{20}$ The NIHSS is frequently used in therapeutic trials of stroke rehabilitation as the primary serial measure of the efficacy of interventions designed to ameliorate sequelae 
TABLE 6. Model-adjusted odds ratios for nonfatal or fatal stroke ( $\mathrm{n}=92$ patients) associated with preoperative risk factors*

\begin{tabular}{lcc}
\hline \multicolumn{1}{c}{ Predictor } & OR $(\mathbf{9 5} \% \mathbf{C I})$ & $\boldsymbol{P}$ value \\
\hline Medical history/preoperative factor & & \\
Neurologic dysfunction & $1.81(1.35-2.42)$ & $<.001$ \\
Carotid disease & $1.75(1.07-2.87)$ & .03 \\
Pulmonary disease & $2.11(1.15-3.88)$ & .02 \\
Pulse pressure (per $10 \mathrm{~mm} \mathrm{Hg}$ & $1.36(1.25-1.48)$ & $<.001$ \\
$\quad$ increment) $\dagger$ & & \\
Redo surgery $\dagger$ & $2.47(1.50-4.08)$ & $<.001$ \\
\hline
\end{tabular}

$O R$, Odds ratio; $C I$, confidence interval. *Excluded were 13 patients with missing values for at least one of the risk factors in the model, including the covariates. †Pulse pressure per $10 \mathrm{~mm} \mathrm{Hg}$ increment above a threshold of $40 \mathrm{~mm} \mathrm{Hg}$. $\ddagger$ Redo surgery was defined as with previous $\mathrm{CABG}$, valve, cardiac, noncardiac, or bypass graft surgery.

after documented stroke. ${ }^{21-23}$ It is often chosen for its simplicity of administration, its high reproducibility of results among allied health personnel, and the ease with which results can be transformed for database collection and statistical analysis. Although previously used in the setting of car- diac surgery to screen for postoperative neurologic changes in patients with and without suspected acute stroke, ${ }^{4-6}$ the NIHSS had not been previously validated as a tool for diagnosing and quantifying the degree of neurologic injury (or lack thereof) in this setting. Our findings indicate that postoperative worsening of the NIHSS score is an excellent predictor of type I outcome after CABG surgery.

The MMSE is a brief, standardized, and validated instrument that was originally used to screen for Alzheimer's disease and is now also used to screen for dementia. ${ }^{7,8,24}$ It is commonly used in both clinical and research settings to measure cognitive impairment in various disease states, document intellectual changes over time, and assess the effects of potential therapeutic agents on cognitive function. The MMSE is attractive because it is brief, easily administered, and easily scored. Estimates of internal consistency ${ }^{25}$ and interrater reliability ${ }^{7,26}$ are good. Premorbid intelligence and educational attainment affect MMSE scores, in that less intelligent or less educated individuals tend to score lower

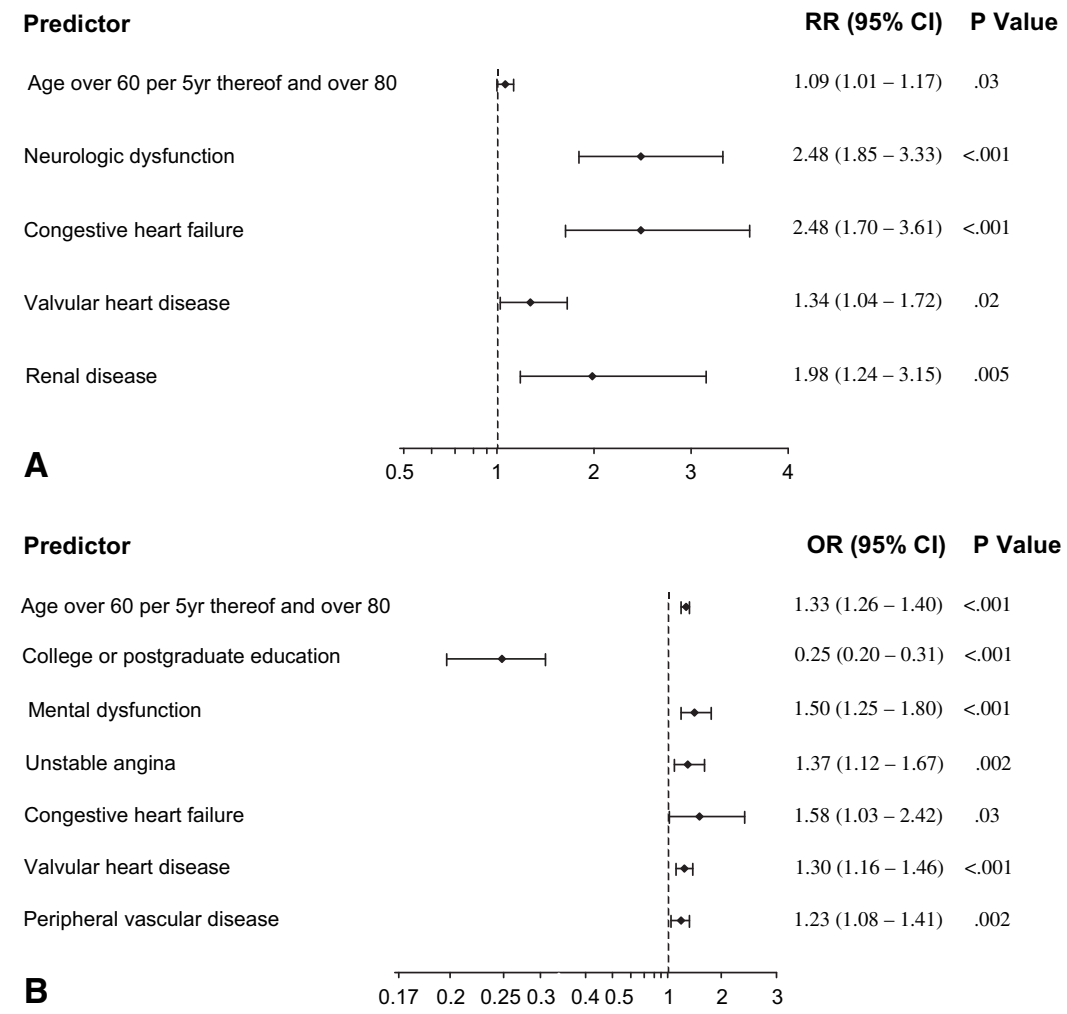

FIGURE 2. Multivariable predictors of adverse preoperative NIHSS or MMSE scores. A, Zero-inflated Negative binomial model for preoperative NIHSS score. A Logit model with binomial assumption is used to determine whether an individual count outcome is from the always-zero or the not-always-zero group, and then a Negative binomial model for count data is performed to model outcomes in the not-always-zero group. For the logistic portion, age increment is related to NIHSS score of zero with a $30 \%$ decrease in odds ( $\mathrm{OR}=0.70 ; 95 \% \mathrm{CI}, 0.55-0.91, P=.008)$; higher education increases the odds to 3 -fold $(\mathrm{OR}=3.56 ; 95 \% \mathrm{CI}, 1.57-8.05, P=.003)$. For the Negative binomial portion, age increment, neurologic dysfunction, congestive heart failure, valvular heart disease, and renal disease increase the risk of increasing NIHSS score (adverse NIHSS score). B, Generalized estimating equations model for ordinal outcome analysis for preoperative MMSE score. MMSE score was categorized as high (27-30), moderate (24-26), and low ( $<24)$. Age increment, mental dysfunction, unstable angina, congestive heart failure, valvular heart disease, and peripheral vascular disease increase the risk of decreasing MMSE score category (adverse MMSE score); higher education decreases the risk of adverse MMSE score. Country was adjusted for in both NIHSS (A) and MMSE (B) models. OR, Odds ratio; $R R$, rate ratio; $C I$, confidence interval. 
than do more intelligent (ie, higher IQ) or more educated individuals. ${ }^{17,25,27}$ Also, MMSE scores decrease with advancing age, from a median score of 29 at 18 to 24 years of age to a median of 25 at $\geq 80$ years of age. ${ }^{24,27}$ Although the MMSE has been used in the setting of cardiac surgery to measure cognitive change in patients with and without suspected changes in intellectual function, ${ }^{4,6,9}$ the preoperative and postoperative clinical utility of MMSE scores has not been published for a large population of surgical patients of any type. Our patients scored relatively well on this test in the preoperative period (median MMSE score of 29 with an interquartile range of 27-30.). Not unexpectedly, we have confirmed that preoperative MMSE scores are related to age and education. Our findings indicate that postoperative worsening of the MMSE score provides only fair discrimination for type I or II outcomes after CABG surgery.

Common predictors of deterioration in NIHSS or MMSE and overt type I or II adverse outcomes included older age, a history of neurologic dysfunction (due to stroke or transient ischemic attack), and diabetes, as well as intraoperative red blood cell transfusion and duration of CPB. These risk factors have been identified as being associated with adverse neurologic outcome. ${ }^{11,28-32}$ If only nonfatal or fatal stroke outcomes are considered, multivariate analysis confirmed some of the same preoperative predictors, notably a history of neurologic dysfunction. These findings may have clinical implications, in that perhaps patients with a known history of stroke or transient ischemic attack should undergo myocardial revascularization in hospitals having immediately available services for the treatment of acute stroke. ${ }^{32}$

In our previous study, ${ }^{11}$ completed in 1993 , we did not formally assess neurologic function with any recognized scale or scoring system in either the preoperative or postoperative periods. In both the previous and the current study, individual investigators at each site assessed type I deficits or type II dysfunction in whatever manner they deemed clinically suitable. Notably, the incidence of type I outcomes in the present study was similar to that in our previous study $(2.9 \%$ vs $3.1 \%)$, whereas the incidence of type II outcomes was somewhat higher in the present study $(6.4 \%$ vs $3.0 \%) .{ }^{11}$ This may be because patients in the current era are older and more likely to have mild preoperative or postoperative cognitive deficits, although, fortunately, advances in cardiac surgical techniques and extracorporeal circulation technology have prevented increases in the incidence of frank stroke. ${ }^{5}$

As in our previous study, ${ }^{11}$ we examined conservative measures of resource use, namely, the duration of intensive care, the total duration of the hospital stay after surgery, and the rate of discharge to intermediate- or long-term care facilities. All 3 measures were markedly prolonged for patients with either type I or type II adverse neurologic outcomes. Compared with patients without adverse neurologic out- comes, patients with type I outcome stayed an additional $2 \frac{1}{2}$ days in the intensive care unit and an additional 5 days on the ward, suggesting that regardless of institutional practice, substantial resources are consumed by such patients. Furthermore, compared with patients without adverse neurologic outcomes, patients with type II outcomes stayed an additional 3 days in the intensive care unit and 3 days on the ward. (These data are included in Table 1.) On the basis of conservative estimates of boarding charges of approximately $\$ 3700$ per day in an intensive care unit and $\$ 1700$ per day on a ward, type I neurologic events are responsible for approximately $\$ 18,000$ in additional costs per patient in in-hospital boarding costs, and type II events are responsible for approximately $\$ 16,000$ in additional costs per patient. Furthermore, the costs of changes in discharge planning and long-term out-of-hospital medical and rehabilitative services undoubtedly result in considerable additional expenditure.

\section{Study Limitations}

Several limitations of the present study may affect the generalizability and utility of its findings. The data are approximately 10 years old, and the patients were only at moderate risk for adverse outcomes. Although overt neurologic adverse events were a relatively small percentage of the total, our incidence of type I outcome $(2.9 \%)$ is not dissimilar from other more recent series. ${ }^{29-31}$ As in our previous study, ${ }^{11}$ although data were collected prospectively, the study is limited by its purely observational design. The study was conducted with data collected from medical centers throughout the world, a diversity that is both a strength and a potential weakness. The assessments were made by multiple investigators who were not neurologists, and the assessments were not always made by the same investigator within an institution. Furthermore, we were not able to consistently obtain objective evidence of acute cerebral ischemic events (eg, computed tomography or magnetic resonance imaging scans) in the postoperative period. In addition, only $87.2 \%$ of patients (4105 of 4707) completed the preoperative and postoperative NIHSS tests, and even fewer completed the MMSE test (2868 of 4707 [60.9\%]). Finally, a complete battery of neurocognitive tests were not administered, only the MMSE. In the absence of more extensive evaluation of neurocognitive dysfunction, we did attempt to be rigorous in identifying type II outcomes, mandating that 2 or more indications of "intellectual dysfunction" be present on 2 or more days of a patient's hospitalization.

Despite these limitations, our current findings warrant additional investigations. Future studies should examine the long-term outcomes associated with unexpected postoperative worsening of either test score. Outcomes of interest would include worsening in patient scores several years after surgery $^{33}$; ability to return to employment; functional outcomes as measured by quality of life assessment tools; 
need for rehospitalization; and postdischarge morbidity due to depression, late stroke, or death.

\section{CONCLUSIONS}

Standardized scores on the NIHSS and MMSE tests are used for the quantitative assessment of adverse neurologic events after cardiac surgery. We have provided a reference defining the clinical utility of assessment of baseline scores and postoperative worsening in scores on these tests, which will be useful in the evaluation of results of clinical trials designed to diagnose, mitigate, or treat adverse neurologic outcomes after cardiac surgery.

The authors are indebted to Stephen N. Palmer, PhD, ELS, for editorial support.

\section{References}

1. Brott T, Adams HP Jr, Olinger CP, Marler JR, Barsan WG, Biller J, et al. Measurements of acute cerebral infarction: a clinical examination scale. Stroke. 1989;20:864-70.

2. Lyden P, Raman R, Liu L, Grotta J, Broderick J, Olson S, et al. NIHSS training and certification using a new digital video disk is reliable. Stroke. 2005;36:2446-9.

3. Duncan PW, Zorowitz R, Bates B, Choi JY, Glasberg JJ, Graham GD, et al. Management of Adult Stroke Rehabilitation Care: a clinical practice guideline. Stroke. 2005;36:e100-43.

4. Mathew JP, Fontes ML, Tudor IC, Ramsay J, Duke P, Mazer CD, et al. A multicenter risk index for atrial fibrillation after cardiac surgery. JAMA. 2004;291:1720-9.

5. Cook DJ, Huston J III, Trenerry MR, Brown RD Jr, Zehr KJ, Sundt TM III. Postcardiac surgical cognitive impairment in the aged using diffusion-weighted magnetic resonance imaging. Ann Thorac Surg. 2007;83:1389-95.

6. Shernan SK, Fitch JC, Nussmeier NA, Chen JC, Rollins SA, Mojcik CF, et al. Impact of pexelizumab, an anti-C5 complement antibody, on total mortality and adverse cardiovascular outcomes in cardiac surgical patients undergoing cardiopulmonary bypass. Ann Thorac Surg. 2004;77:942-9.

7. Folstein MF, Folstein SE, McHugh PR. "Mini-mental state": a practical method for grading the cognitive state of patients for the clinician. J Psychiatr Res. 1975; 12:189-98.

8. Hensel A, Angermeyer MC, Riedel-Heller SG. Measuring cognitive change in older adults: reliable change indices for the Mini-Mental State Examination. J Neurol Neurosurg Psychiatry. 2007;78:1298-303.

9. Fitch JC, Rollins S, Matis L, Alford B, Aranki S, Collard CD, et al. Pharmacology and biological efficacy of a recombinant, humanized, single-chain antibody C5 complement inhibitor in patients undergoing coronary artery bypass graft surgery with cardiopulmonary bypass. Circulation. 1999;100:2499-506.

10. Weissrock S, Levy F, Balabaud V, Thiranos JC, Dupeyron JP, Steib A. [Interest of the Mini Mental State Examination to detect cognitive defects after cardiac surgery]. Ann Fr Anesth Reanim. 2005;24:1255-61.

11. Roach GW, Kanchuger M, Mangano CM, Newman M, Nussmeier N, Wolman R, et al. Adverse cerebral outcomes after coronary bypass surgery. Multicenter Study of Perioperative Ischemia Research Group and the Ischemia Research and Education Foundation Investigators. N Engl J Med. 1996;335:1857-63.

12. Mangano DT. Aspirin and mortality from coronary bypass surgery. $N$ Engl J Med. 2002;347:1309-17.
13. Goldstein LB, Bertels C, Davis JN. Interrater reliability of the NIH stroke scale. Arch Neurol. 1989;46:660-2.

14. Goldstein LB, Samsa GP. Reliability of the National Institutes of Health Stroke Scale: extension to non-neurologists in the context of a clinical trial. Stroke. 1997;28:307-10.

15. Vickers AJ, Altman DG. Statistics notes: analyzing control trials with baseline and follow-up measurements. BMJ. 2001;323:1123-4.

16. O'Bryant SE, Humphreys JD, Smith GE, Ivnik RJ, Graff-Radford NR, Petersen RC, et al. Detecting dementia with the mini-mental state examination in highly educated individuals. Arch Neurol. 2008;65:963-7.

17. Tombaugh TN, McIntyre NJ. The mini-mental state examination: a comprehensive review. J Am Geriatr Soc. 1992;40:922-35.

18. Lyden P, Lu M, Jackson C, Marler J, Kothari R, Brott T, et al. Underlying structure of the National Institutes of Health Stroke Scale: results of a factor analysis. NINDS tPA Stroke Trial Investigators. Stroke. 1999;30:2347-54.

19. Tong DC, Yenari MA, Albers GW, O'Brien M, Marks MP, Moseley ME. Correlation of perfusion and diffusion-weighted MRI with NIHSS score in acute $(<6.5$ hour) ischemic stroke. Neurology. 1998;50:864-70.

20. Wkhloo AK, Sandhu JS. Principles of endovascular therapy. In: Bradley WG, Daroff RB, Fenichel GM, Jancovic J, eds. Neurology in clinical practice: principles of diagnosis and management. 4th ed. Philadelphia: Elsevier; 2004:993-1025.

21. Adams HP Jr, Davis PH, Leira EC, Chang KC, Bendixen BH, Clarke WR, et al. Baseline NIH Stroke Scale score strongly predicts outcome after stroke: a report of the Trial of Org 10172 in Acute Stroke Treatment (TOAST). Neurology. 1999;53: 126-31.

22. Young FB, Weir CJ, Lees KR. Comparison of the National Institutes of Health Stroke Scale with disability outcome measures in acute stroke trials. Stroke. 2005;36:2187-92.

23. Bruno A, Saha C, Williams LS. Using change in the National Institutes of Health Stroke Scale to measure treatment effect in acute stroke trials. Stroke. 2006;37: 920-1.

24. Spreen O, Strauss E. A compendium of neuropsychological tests: administration, norms, and commentary. 2nd ed. New York: Oxford University Press; 1998.

25. Tombaugh TN, McDowell I, Kristjansson B, Hubley AM. Mini-Mental State Examination (MMSE) and the Modified MMSE (3MS): a psychometric comparison and normative data. Psychol Assess. 1996;8:48-59.

26. Foster JR, Sclan S, Welkowitz J, Boksay I, Seeland I. Psychiatric assessment in medical long-term care facilities: reliability of commonly used rating scales. Int J Geriatr Psychiatry. 1988;3:229-33.

27. Crum RM, Anthony JC, Bassett SS, Folstein MF. Population-based norms for the Mini-Mental State Examination by age and educational level. JAMA. 1993;269: 2386-91.

28. Baker R, Hallsworth LJ, Knight RB. Stroke after coronary artery bypass grafting. Ann Thorac Surg. 2005;80:1746-50.

29. Anyanwu AC, Filsoufi F, Salzberg SP, Bronster DJ, Adams DH. Epidemiology of stroke after cardiac surgery in the current era. J Thorac Cardiovasc Surg. 2007; 134:1121-7.

30. Lisle TC, Barrett KM, Gazoni LM, Swenson BR, Scott CD, Kazemi A, et al. Timing of stroke after cardiopulmonary bypass determines mortality. Ann Thorac Surg. 2008;85:1556-63.

31. Salis S, Mazzanti VV, Merli G, Salvi L, Tedesco CC, Veglia F, et al. Cardiopulmonary bypass duration is an independent predictor of morbidity and mortality after cardiac surgery. J Cardiothorac Vasc Anesth. 2008;22:814-22.

32. Dafer RM. Risk estimates of stroke after coronary artery bypass graft and carotid endarterectomy. Neurol Clin. 2006;24:795-806.

33. Selnes OA, Grega MA, Bailey MAM, Pham LD, Zeger SL, Baumgartner WA, et al. Cognition 6 years after surgical or medical therapy for coronary artery disease. Ann Neurol. 2008;63:581-90. 


\section{Appendix 1.}

The IREF is an independent nonprofit foundation, formed in 1987, that develops clinical investigators via observational studies and clinical trials addressing ischemic injury of the heart, brain, kidney, and gastrointestinal tract. The IREF provided all funding for execution of the study, collection of the data, and analysis and publication of the findings. The Multicenter Study of Perioperative Ischemia Research Group, formed in 1988, is an association of 160 international medical centers located in 23 countries organized through and supported by grants from the IREF.

The following institutions and persons coordinated the Multicenter Study of Perioperative Ischemia Research Group EPI-II study. Study Chairman-D. Mangano; Senior Editors-J. Levin, L. Saidman; Study Design and Analysis Center: Ischemia Research and Education FoundationP. Barash, C. Dietzel, A. Herskowitz, K. Huang, Y. Miao, I. C. Tudor, S. Wang, Y. Weng; Editorial/Administrative Group-D. Beatty, I. Lei, B. Xavier.

The following institutions and persons participated in the McSPI EPI-II Study. Centers and investigators: United States-University of Chicago, Weiss Memorial Hospital-S. Aronson; Beth Israel Hospital-M. Comunale; Massachusetts General-M. D'Ambra; University of Rochester - M. Eaton; Baystate Medical Center-R. Engelman; Baylor College of Medicine-J. Fitch; Duke Medical Center-K. Grichnik; UTHSCSA-Audie Murphy VA, UTHSCSA-University Hospital-C. B. Hantler; St. Luke's Roosevelt Hospital-Z. Hillel; New York University Medical Center-M. Kanchuger, J. Ostrowski; Stanford University Medical Center-C. M. Mangano; Yale University School of Medicine-J. Mathew, M. Fontes, P. Barash; University of Wisconsin-M. McSweeney, R. Wolman; University of Arkansas for Medical Sciences-C. A. Napolitano; Discovery Alliance, Inc.-L. A. Nesbitt; VA Medical Center, Milwaukee-N. Nijhawan; Texas Heart Institute, Mercy Medical Center-N. Nussmeier; University of Texas Medical School, Houston-E. G. Pivalizza; University of Arizona-S. Polson; Emory University Hospital-J. Ramsay; Kaiser Foundation Hospital-G. Roach; Thomas Jefferson University Hospital, MCP Hahnemann University Hospital- N. Schwann; VAMC Houston-S. Shenaq; Maimonides Medical Center-K. Shevde; Mt. Sinai Medical Center-L. Shore-Lesserson, D. Bronheim; University of Michigan - J. Wahr; University of Washington-B. Spiess, I. Wright; VA Medical Center, S. F.-A. Wallace; Austria-University of Graz-H. Metzler; Canada-University of British Columbia-D. Ansley, J. P. O'Connor; The Toronto Hospital-D. Cheng; Laval Hospital, Quebec-D. Côte; Health Sciences Centre-University of Manitoba-P. Duke; University of Ottawa Heart InstituteJ. Y. Dupuis, M. Hynes; University of Alberta Hospital—B. Finegan; Montreal Heart Institute-R. Martineau, P. Cou- ture; St. Michael's Hospital, University of Toronto-D. Mazer; Colombia-Fundacion Clinico Shaio-J. C. Villalba, M. E. Colmenares; France-CHRU Le Bocage-C. Girard; Hospital Pasteur-C. Isetta; Germany-Universität Wrzburg-C. A. Greim, N. Roewer; Universität BonnA. Hoeft; University of Halle-R. Loeb, J. Radke; Westfalische Wilhelms-Universität Munster-T. Mollhoff; Universität Heidelberg-J. Motsch, E. Martin; LudwigMaximillians Universität-E. Ott; Universität Krankenhaus Eppendorf-J. Scholz, P.Tonner; Georg-August Universität Göttingen-H. Sonntag; Ludwig-Maximillians Universität (Department of Cardiac Surgery)-P. Ueberfuhr; Hungary-Orszagos Kardiologiai Intezet—A. Szekely; IndiaEscorts Heart Institute-R. Juneja; Apollo Hospital-G. Mani; Israel—Hadassah University Hospital—B. Drenger, Y. Gozal, E. Elami; Italy—San Raffaele Hospital, Universita de Milano-C. Tommasino; Mexico-Instituto Nacional de Cardiologia—P. Luna; The Netherlands-University Hospital Maastricht—P. Roekaerts, S. DeLange; Poland—Institute of Cardiology-R. Pfitzner; Romania-Institute of Cardiology—D. Filipescu; Thailand-Siriraj Hospital-U. Prakanrattana; United Kingdom-Glenfield Hospital—D. J. R. Duthie; St. Thomas' Hospital—R. O. Feneck; The Cardiothoracic Centre, Liverpool-M. A. Fox; South Cleveland Hospital—J. D. Park; Southampton General Hospital—D. Smith; Manchester Royal Infirmary-A. Vohra; Papworth Hospital-A. Vuylsteke, R. D. Latimer.

Appendix 2. Demographic characteristics

Age, or age $>60$ y and per 5 y thereof and $>80 \mathrm{y}$
Gender
Obesity (BMI $>28$ )
Regular or current smoke
College or postgraduate education
Race or ethnicity
Medical history or preoperative factors
Stroke
TIA
Preoperative neurologic dysfunction (history of stroke or TIA)
Preoperative mental dysfunction
Myocardial infarction
Unstable angina
Congestive heart failure
Hypertension
Pulse pressure
Controlled hypertension
Uncontrolled hypertension
Atrial fibrillation/atrial flutter
Dysrhythmia
Tachyarrhythmias
Cardiomegaly abnormality (preoperative chest x-ray)
Valvular stenosis
Valvular insufficiency


Appendix 2. Continued

Preoperative valve (aortic/mitral) calcification: chest $\mathrm{x}$-ray/cardiac catheterization

Any valve (aortic/mitral) disease (stenosis/insufficiency/calcification) on admission or

Preoperation

Redo surgery

Preoperative IABP

Preoperative aortic vascular disease

Peripheral vascular disease

Carotid vascular disease

Pulmonary disease

Diabetes

Renal disease

Liver disease

Hematologic disorder

Previous carotid endarterectomy

Intraoperative and surgical factors

Concurrent combined surgery

Bypass of $>3$ proximal aortic anastomoses
Appendix 2. Continued

Aortic vascular disease (history or preoperative or intraoperative image/ palpation)

All vascular disease, carotid vascular disease, peripheral vascular disease, or aortic vascular disease (history, preoperative, or intraoperative image/ palpation)

Valve stenosis (history, preoperative, or prebypass)

Valve insufficiency (history, preoperative, or prebypass)

Valvular calcification (preoperative or intraoperative)

Any valve (aortic/mitral) disease (stenosis/insufficiency/calcification)

(on admission or preoperative or intraoperative)

Intraoperative hypertension

Intraoperative atrial fibrillation/atrial flutter

Intraoperative homologous red blood cell transfusion

Intraoperative homologous fresh-frozen plasma transfusion

Intraoperative homologous platelets transfusion

Return to $\mathrm{CPB}$

CPB time (min)

$B M I$, Body mass index; TIA, transient ischemic attack; IABP, intraaortic balloon pump; $C P B$, cardiopulmonary bypass.

Appendix 3. Receiver operating characteristic curves, sensitivity, and specificity of National Institutes of Health Stroke Scale score by postoperative day of National Institutes of Health Stroke Scale test

\begin{tabular}{|c|c|c|c|c|c|}
\hline CNS type I* outcome & POD of NIHSS test & Test of NIHSS & ROC curve & Specificity & Sensitivity \\
\hline Type I & POD3 & NIHSS change $\geq 1$ & 0.87 & 0.88 & 0.86 \\
\hline Type I & POD3 & NIHSS change $\geq 2$ & 0.79 & 0.96 & 0.62 \\
\hline Type I & POD3 & NIHSS change $\geq 3$ & 0.75 & 0.98 & 0.52 \\
\hline Type I & POD3 & NIHSS change $\geq 4$ & 0.73 & 0.98 & 0.48 \\
\hline Type I & POD4 & NIHSS change $\geq 1$ & 0.82 & 0.88 & 0.76 \\
\hline Type I & POD4 & NIHSS change $\geq 2$ & 0.80 & 0.95 & 0.65 \\
\hline Type I & POD4 & NIHSS change $\geq 3$ & 0.72 & 0.98 & 0.47 \\
\hline Type I & POD4 & NIHSS change $\geq 4$ & 0.70 & 0.99 & 0.41 \\
\hline Type I & POD5 & NIHSS change $\geq 1$ & 0.84 & 0.85 & 0.83 \\
\hline Type I & POD5 & NIHSS change $\geq 2$ & 0.84 & 0.93 & 0.75 \\
\hline Type I & POD5 & NIHSS change $\geq 3$ & 0.77 & 0.96 & 0.58 \\
\hline Type I & POD5 & NIHSS change $\geq 4$ & 0.78 & 0.98 & 0.58 \\
\hline
\end{tabular}

CNS, Central nervous system; POD, postoperative day; NIHSS, National Institutes of Health Stroke Scale; ROC, receiver operating characteristic. *Type I, event(s) before postoperative NIHSS test or MMSE test, or type I-neurologic death.

Appendix 4. Receiver operating characteristic curves, sensitivity, and specificity of Mini-Mental State Examination score by postoperative day of Mini-Mental State Examination test

\begin{tabular}{|c|c|c|c|c|c|}
\hline CNS type I* outcome & POD of MMSE Test & Test of NIHSS & ROC curve & Specificity & Sensitivity \\
\hline Type I & POD3 & MMSE change $\leq-1$ & 0.61 & 0.55 & 0.67 \\
\hline Type I & POD3 & MMSE change $\leq-2$ & 0.64 & 0.73 & 0.54 \\
\hline Type I & POD3 & MMSE change $\leq-3$ & 0.67 & 0.84 & 0.50 \\
\hline Type I & POD3 & MMSE change $\leq-4$ & 0.68 & 0.91 & 0.46 \\
\hline Type I & POD4 & MMSE change $\leq-1$ & 0.68 & 0.56 & 0.80 \\
\hline Type I & POD4 & MMSE change $\leq-2$ & 0.65 & 0.75 & 0.55 \\
\hline Type I & POD4 & MMSE change $\leq-3$ & 0.65 & 0.85 & 0.45 \\
\hline Type I & POD4 & MMSE change $\leq-4$ & 0.63 & 0.91 & 0.35 \\
\hline Type I & POD5 & MMSE change $\leq-1$ & 0.72 & 0.53 & 0.92 \\
\hline Type I & POD5 & MMSE change $\leq-2$ & 0.72 & 0.72 & 0.71 \\
\hline Type I & POD5 & MMSE change $\leq-3$ & 0.75 & 0.83 & 0.67 \\
\hline Type I & POD5 & MMSE change $\leq-4$ & 0.76 & 0.89 & 0.63 \\
\hline
\end{tabular}

$C N S$, Central nervous system; ROC, receiver operating characteristic; POD, postoperative day; NIHSS, National Institutes of Health Stroke Scale; $M M S E$, Mini-Mental State Examination. *Type I, event(s) before postoperative NIHSS test or MMSE test, or type I-neurologic death. 